\title{
Research on large inertia vehicle's handling stability based on improved evidence theory
}

\author{
Yajun Shao, Wei Zhang, Qinhe Gao, Hongjie Cheng, Yong Jia, Xingyu Xie \\ Xian Research Institute of Hi-Tech, Shaanxi 710025, China \\ yajun_wo@163.com
}

\begin{abstract}
Key words: Vehicle with large inertia; handling stability evaluation; objective weighting; improved evidence theory

Abstract. The objective evaluation index system of large inertia vehicle's handling and stability was set up, the vehicle experiment was performed to get data of evaluation indexes referring to national standard. Three objective weighting methods with different focus were chosen to get the weighting coefficient of each index. An improved D-S evidence theory was raised to fuse three objective weighting methods getting the final index weighting, and the result was compared with the result gotten from subjective evaluation. Finally, it was proved that the improved method presented in this paper can better solve the conflict between three methods, and obtained the reliable index weighting coefficient.
\end{abstract}

\section{Introduction}

Handling and stability performance is one of the most important properties for vehicle's safety[1], which will directly influence the operation state of large inertia vehicle. So it's the basis of ensuring safety and improving execution efficiency of large inertia vehicle to evaluate the handling and stability performance reasonably. There are already detailed provisions on handling and stability evaluation of common double-axle vehicle in national standards, whereas for multi-axle vehicle, it is still blank. Researchers mostly evaluated large inertia vehicle referring to common vehicle's evaluation combining with the large inertia vehicle's real characteristics, whose main idea was reducing the dimension and assigning the weighting of multiple index[2][3], methods such as multi-variate statistical analysis, analytic hierarchy process [4]-[7] were commonly used. But the index system acquired by single method was easy to ignore some important information, leading to one-sided, even opposite results. In this paper, we have extracted eight indexes for large inertia vehicle's evaluation based on road experiment of large inertia vehicle and the vehicle's real characteristic, obtained the objective weighting coefficient by using three different methods according to the statistical decision theory of multi-index evaluation. Finally, the final index system was obtained through information fusion by an improved D-S evidence theory. The final result's credibility was verified by subjective analytic hierarchy process.

\section{Method of objective weighting}

Considering the excessive test projects and evaluation indexes of vehicles, the high security requirement and high difficulty of road test, this paper used three different objective weighting methods to get index weight, which could obtain as much information as possible with less indexes and could ensure the accuracy and credibility of the evaluation, the methods were variation coefficient method, entropy method, correlation coefficient method respectively[8][9].

The variation coefficient method assigned the weight of each index according to the variation 
degree of observed value on all evaluated objects. The basic idea of this method is the variation degree affects the final evaluation result directly, the normalized value of variation coefficient is generally considered as the weighting coefficient.

Supposing the number of evaluated index was $a$, each index corresponded to $b$ different objects. $X$ was the original matrix of index values, $x_{i j}$ standed for the $j^{\text {th }}$ index value of the $i^{\text {th }}$ object, then

$$
X=\left[\begin{array}{lll}
x_{11} & x_{12} \mathrm{~L} & x_{1 a} \\
x_{21} & x_{22} \mathrm{~L} & x_{2 a} \\
\mathrm{M} & \mathrm{ML} & \mathrm{M} \\
x_{b 1} & x_{b 2} \mathrm{~L} & x_{b a}
\end{array}\right]
$$

Calculate the standard deviation of each index firstly, $S_{j}$, which reflects the variation degree;

then calculate the variation coefficient reflecting relative variation degree of each index, $V_{j}$.

$$
v_{j}=\frac{S_{j}}{\bar{x}_{j}}
$$

The weighting coefficient of each index, $w_{j 1}$, can be obtained by normalizing the variation coefficient.

$$
w_{j 1}=\frac{v_{j}}{\sum_{j=1}^{a} v_{j}}
$$

In order to highlight the importance of the variation degree on the evaluation result, the entropy method was selected as the auxiliary of the variation method. The entropy method assigned the weight of each index according to the amount of information each index delivering. The more information the index delivers, the smaller the uncertainty and the entropy is. The entropy of evaluation index could be expressed by the following equation:

$$
H_{j}=-k \sum_{i=1}^{b} M_{i j} \ln \left(M_{i j}\right)
$$

Where $M_{i j}$ was the normalized index value, $k=1 / \ln b, i=1,2,3, \mathrm{~L}, b, j=1,2,3, \mathrm{~L}, a$. The index weight could be calculated by the following equation:

$$
w_{j 2}=\frac{1-H_{j}}{a-\sum_{j=1}^{a} H_{j}}
$$

The principle of entropy method was similar to the variation coefficient method, the index weighting coefficient could not reflect the data s independence. So this paper leaded in an assigning method considering the data's independence to make up for the information loss caused by the above two methods, called multiple correlation coefficient method. This method assigned the weight of each index according to information's multiplicity and index's independence, the smaller the 
multiplicity is and the more independent the index is, the bigger the weighting coefficient is. The correlation matrix of all indexes, $R$, would be obtained firstly, in order to calculate the multiple correlation coefficient matrix between the $n^{\text {th }}$ index $X_{n}$ and the other $n-1$ indexes, decomposed $R$ as the following form:

$$
R=\left[\begin{array}{ll}
R_{n-1} & r_{n} \\
r_{n}^{\prime} & 1
\end{array}\right]
$$

Where $R_{n-1}$ is the correlation coefficient matrix of the other $n-1$ indexes, $r_{n}$ is an $n-1$ order column vector, $r_{n}=\left(r_{1 n}, r_{2 n}, \mathrm{~L}, r_{(n-1) n}\right)$, then the multiple correlation coefficient of $X_{n}$ to other index is

$$
\rho_{n}=r_{n}^{\prime} R_{n-1}^{-1} r_{n}
$$

The weighting coefficient could be obtained by normalizing the reciprocal of the multiple correlation coefficient.

$$
w_{j 3}=\frac{1}{\rho_{j}} / \sum_{j=1}^{a} \frac{1}{\rho_{j}}
$$

Respectively, the above three methods, aiming at different information feature, obtained the weighting coefficient of each index according to different assigning regulations. Each method had its both superiority and one-sideness, conflict and repetition may exist between different methods, causing great uncertainty to final result. So this paper proposed an improved D-S evidence theory to fuse the three results.

\section{Improvement of D-S evidence}

D-S evidence theory is the abbreviation of Dempster-Shafer theory, which was proposed at 1967 by Dempster and popularized at 1978 by Shafer. The basis of evidence theory is pooled evidence and renewing belief function, it describes the uncertainty by using recognition framework, basic probability distribution function, belief function and plausibility function[10][11].

Supposing $\Theta=\left\{\theta_{1}, \theta_{2}, \mathrm{~L}, \theta_{i}, \mathrm{~L}, \theta_{n}\right\}$, then a set of complete states $\Theta$ of disjoint event is the recognition framework. The set made up of whole subsets of $\Theta$ is called power set, expressed as $2^{\ominus}$, standing for all possible propositions set, as the following equation, where $\Phi$ is empty set.

$$
\begin{aligned}
2^{\Theta}= & \left\{\Phi,\left\{\theta_{1}\right\}, \mathrm{L},\left\{\theta_{n}\right\},\left\{\theta_{1}, \theta_{2}\right\},\left\{\theta_{1}, \theta_{3}\right\}, \mathrm{L},\right. \\
& \left.\left\{\theta_{1}, \theta_{n}\right\}, \mathrm{L},\left\{\theta_{n-1}, \theta_{n}\right\}, \mathrm{L}, \Theta\right\}
\end{aligned}
$$

Definition 1: $\Theta$ is the recognition framework, $m$ is the mapping from $2^{\Theta}$ to $[0,1], A$ stands for any subset of $\Theta$, if meeting the conditions: $m(\Phi)=0, \sum_{A \subseteq \Theta} m(A)=1$, then $m$ is regarded as basic probability distribution function of $\Theta . m(A)$ is called the basic reliability of $A$, as also as the mass function of proposition, built according to researchers' experience or detected data. $m(A)$ reflects the level of reliability to $A$. 
Definition 2: $\Theta$ is the recognition framework, $\mathrm{Bel}$ is the mapping from $2^{\Theta}$ to $[0,1]$, if meeting the conditions: $\operatorname{Bel}(A)=\sum_{B \subseteq A, A \subseteq \Theta} m(B)$, then Bel is regarded as belief function of $A$, standing for the degree that the evidence supports $A$. Because Bel could not reflect the degree that the evidence does not supports $A$, the concept of plausibility function is leaded in.

Definition 3: $\Theta$ is the recognition framework, $P l$ is the mapping from $2^{\Theta}$ to $[0,1]$, if meeting the conditions: $P l(A)=1-\operatorname{Bel}(\bar{A})=\sum_{\substack{B \cap A \neq \Phi \\ A, B \subseteq \Theta}} m(B)$, then $P l(A)$ is regarded as the plausibility function of $A$.

Supposing $Q_{1}$ and $Q_{2}$ were two evidence sets of recognition framework $\Theta, m_{1}$ and $m_{2}$ were mass function of the two evidence sets, $A_{i}$ and $B_{j}$ were corresponding focal elements. If $m_{1}$ and $m_{2}$ were not complete conflict, a new mass function could be obtained by improved D-S synthetic rule. The D-S synthetic rule could be defined as:

$$
m(A)=\left\{\begin{array}{cc}
\frac{1}{1-K} \sum_{A_{i} \cap B_{j}=A} m_{1}\left(A_{i}\right) m_{2}\left(B_{j}\right) & A \neq \Phi \\
0 & A=\Phi
\end{array}\right.
$$

Where $K=\sum_{A_{i} \cap B_{j}=\Phi} m_{1}\left(A_{i}\right) m_{2}\left(B_{j}\right)$, the value of $K$ reflects the degree of evidence conflict. The coefficient, $1 /(1-K)$, is called regularization factor.

Evidence theory can solve the low conflict problem by simple reasoning and get preferable result. Whereas the basic probability assignment functions between different evidences encounter high conflict, the synthesis results may be unreasonable, even perverse. This paper can escape and eliminate the unreasonableness by weighting the source data of each evidence. In reference [12], Liu proposed the index, pignistic probability distance and conflict coefficient $K$, to judge the conflict level of evidence, but the index were neither measurable. We proposed a combination rule of evidence theory based on distance according to Liu's idea.

Supposing there are $\mathrm{n}$ kinds of evidence. Firstly, calculate the pignistic probability distance, $\operatorname{difBetP}_{m_{i}}^{m_{j}}$, between the two evidences $\left(m_{i}\right.$ and $\left.m_{j}(i, j=1,2, \mathrm{~L}, n)\right)$, the matrix of pignistic probability distance, $M_{p}$, would be obtained:

$$
M_{p}=\left[\begin{array}{cccc}
0 & \operatorname{difBetP}_{m_{1}}^{m_{2}} & \mathrm{~L} & \operatorname{difBetP} m_{m_{1}}^{m_{n}} \\
\operatorname{difBetP}_{m_{2}}^{m_{1}} & 0 & \mathrm{~L} & \operatorname{difBetP}_{m_{2}}^{m_{n}} \\
\mathrm{M} & \mathrm{M} & \mathrm{O} & \mathrm{M} \\
\operatorname{difBetP}_{m_{n}}^{m_{1}} & \operatorname{difBetP}_{m_{n}}^{m_{2}} & \mathrm{~L} & 0
\end{array}\right]
$$

$$
\begin{aligned}
\operatorname{difBetP}_{m_{1}}^{m_{2}} & =\max _{A \subseteq \Theta}\left(\left|\operatorname{BetP}_{m 1}(A)-\operatorname{BetP}_{m 2}(A)\right|\right) \\
\operatorname{BetP}_{m}(A) & =\sum_{\omega \subseteq A} \operatorname{BetP}_{m}(\omega)
\end{aligned}
$$

$\operatorname{BetP}_{m}(\omega)$ is the pignistic probability function, 
$\left\{\begin{array}{l}\operatorname{BetP}_{m}(\omega)=\sum_{A \subseteq \Theta, \omega \in \mathrm{A}} \frac{1}{|A|} \frac{m(A)}{1-m(\Phi)} \\ m(\Phi) \neq 1\end{array}\right.$

In equation 12, $|A|$ is the number of element in set $\mathrm{A}$.

Then calculate the conflict coefficient, $K_{i j}$, between $m_{i}$ and $m_{j}$. The conflict coefficient matrix, $M_{k}$, would be obtained,

$$
M_{k}=\left[\begin{array}{cccc}
K_{11} & K_{12} & \mathrm{~L} & K_{1 n} \\
K_{21} & K_{22} & \mathrm{~L} & K_{2 n} \\
\mathrm{M} & \mathrm{M} & \mathrm{O} & \mathrm{M} \\
K_{n 1} & K_{n 2} & \mathrm{~L} & K_{n n}
\end{array}\right]
$$

$K=\sum_{A_{i} \cap B_{j}=\Phi} m_{1}\left(A_{i}\right) m_{2}\left(B_{j}\right)$

Based on $M_{p}$ and $M_{k}$, we describe $k p_{i j}\left(k p_{i j}=\left(K_{i j}, \operatorname{difBetP}_{m_{i}}^{m_{j}}\right)\right)$ as a point in the $K$-difBetP plane. Define $d_{i j}$ as the conflict distance,

$$
d_{i j}=\left|\begin{array}{l}
k_{i j} \\
k p_{i j}
\end{array}\right|=\sqrt{K_{i j}^{2}+\left(\operatorname{difBetP}_{m_{i}}^{m_{j}}\right)^{2}}
$$

Define $s_{i j}$ is the similarity level between $m_{i}$ and $m_{j}$.

$$
s_{i j}=1 / d_{i j}
$$

The similarity level matrix, $S_{M}$, would be obtained,

$$
S_{M}=\left[\begin{array}{cccc}
s_{11} & s_{12} & \mathrm{~L} & s_{1 n} \\
s_{21} & s_{22} & \mathrm{~L} & s_{2 n} \\
\mathrm{M} & \mathrm{M} & \mathrm{O} & \mathrm{M} \\
s_{n 1} & s_{n 2} & \mathrm{~L} & s_{n n}
\end{array}\right]
$$

The support degree of evidence $m_{i}$ is:

$$
\sup \left(m_{i}\right)=\sum_{\substack{j=1 \\ i \neq j}}^{n} S_{i j}
$$

The weight coefficients could be determined by normalizing the support degree of evidence.

$$
\omega_{i}=\frac{\sup \left(m_{i}\right)}{\sum_{i=1}^{n} \sup \left(m_{i}\right)}
$$

We can weighting the source data by using $\omega_{i}$, and the final result could be obtained by fusing the new data according to the synthesis rule of evidence theory. 


\section{Example of large inertia vehicle evaluation}

A four-axle vehicle was chosen as the research object in this paper. According to the national standard[13] and the real characteristics of the vehicle, the steady state circular test, the cornering and self-aligning test, the steering wheel angle pulse test were performed to evaluate the vehicle's handling performance. There were eight evaluation indexes: the lateral acceleration at neutral, $a_{n}$; the under steer rate, $U$; the roll rate of the vehicle body, $K_{\phi}$; the yaw rate s absolute value, $\Delta r$; the yaw rate s total variance, $E_{r}$, reminded after loosing the wheel $3 \mathrm{~s}$; the resonant frequency, $f$; the resonance peak degree, $D$; the phase lag angle, $\alpha$. We tested eight large inertia vehicles and obtained results of the test, shown in table 1 . The first and the last vehicles were virtual vehicles, whose data were corresponding to the lower and the upper limit value of the indexes in national standard.

Table1. Test data of indexes

\begin{tabular}{ccccccccc}
\hline & $a_{n} /\left[\mathrm{m} / \mathrm{s}^{2}\right]$ & $U /\left[\left(\mathrm{g} /\left(\mathrm{m} / \mathrm{s}^{2}\right)\right]\right.$ & $K_{\phi} /\left[\left(\mathrm{g} /\left(\mathrm{m} / \mathrm{s}^{2}\right)\right]\right.$ & $f_{F} / \mathrm{Hz}$ & $D / \mathrm{dB}$ & $\alpha /(\mathrm{o})$ & $\Delta r /[(\mathrm{o}) / \mathrm{s}]$ & $E_{r} / \mathrm{s}$ \\
\hline veh01 & 3 & 1.2 & 1.4 & 0.3 & 5 & 100 & 6 & 0.9 \\
veh02 & 3.10 & 0.58 & 0.31 & 2.85 & 1.58 & 91.1 & 0.62 & 0.65 \\
veh03 & 4.32 & 0.46 & 0.96 & 4.02 & 3.28 & 76.5 & 2.11 & 0.67 \\
veh04 & 6.32 & 0.96 & 0.15 & 1.93 & 3.01 & 95.1 & 3.22 & 0.83 \\
veh05 & 5.29 & 0.52 & 1.21 & 1.37 & 3.19 & 93.6 & 1.87 & 0.62 \\
veh06 & 5.39 & 0.23 & 1.02 & 1.28 & 3.9 & 86.5 & 4.73 & 2.03 \\
veh07 & 4.47 & 0.46 & 1.19 & 1.47 & 5.56 & 88.4 & 2.38 & 0.31 \\
veh08 & 6 & 0.5 & 0.7 & 0.5 & 2 & 60 & 0 & 0.45
\end{tabular}

The test data in table 1 were processed by three different methods introduced in chapter 1, three groups of index weighting coefficients were obtained, shown in table 2 .

Table2. Results of objective weighting

\begin{tabular}{ccccccccc}
\hline & $a_{n}$ & $U$ & $K_{\phi}$ & $f_{F}$ & $D$ & $\alpha$ & $\Delta r$ & $E_{r}$ \\
\hline $\begin{array}{c}\text { variation coefficient } \\
\text { method }\end{array}$ & 0.07 & 0.13 & 0.13 & 0.18 & 0.10 & 0.04 & 0.19 & 0.16 \\
$\begin{array}{c}\text { entropy method } \\
\text { correlation coefficient }\end{array}$ & 0.03 & 0.10 & 0.14 & 0.21 & 0.07 & 0.01 & 0.28 & 0.15 \\
$\quad 0.46$ & 0 & 0.10 & 0.09 & 0.05 & 0.02 & 0.17 & 0.10 \\
\hline method & & & & & & & &
\end{tabular}

As shown in table 2, there existed high conflict between three different methods. To investigate the synthesis effect of this paper's method, data fusion was made by three different methods: taking the average value, basic evidence theory and improved method of this paper. The subjective evaluation considering human-vehicle factor was also performed by analytic hierarchy process shown in reference [6].

When evaluated subjectively, three experienced drivers of large inertia vehicle scored the vehicle, the average of them three was the subjective result. The fused results and the subjective result were shown in table 3 . 
Table3. Results of data fusion and subjective evaluation

\begin{tabular}{ccccccccc}
\hline & $a_{n}$ & $U$ & $K_{\phi}$ & $f_{F}$ & $D$ & $\alpha$ & $\Delta r$ & $E_{r}$ \\
\hline average value & 0.19 & 0.08 & 0.12 & 0.16 & 0.07 & 0.02 & 0.21 & 0.14 \\
basic evidence theory & 0.05 & 0 & 0.10 & 0.19 & 0.02 & 0 & 0.50 & 0.13 \\
$\begin{array}{c}\text { method of this paper } \\
\text { analytic hierarchy }\end{array}$ & 0.13 & 0.06 & 0.11 & 0.20 & 0.04 & 0.05 & 0.32 & 0.09 \\
$\begin{array}{c}\text { process } \\
\text { pan }\end{array}$ & 0.14 & 0.05 & 0.09 & 0.22 & 0.05 & 0.07 & 0.30 & 0.09 \\
\hline
\end{tabular}

As shown in table 3 , the methods of taking average value and basic D-S evidence theory were greatly different in sorting the importance of all indexes and assigning the weight of the indexes, neither of them solved the conflict between those three weighting methods. Both of the methods weakened some indexes in importance compared with the result of subjective evaluation, great difference existed between the results of objective evaluation and human's feeling. The synthesis result of improved evidence theory was similar with the subjective evaluation at sorting the importance of all indexes, so did the index weighting assignment. The improved method of this paper solved the conflict problem reasonably. Thus, the improved synthesis rules could solve the conflict between different weighting methods and make a reasonable judgment on the weighting of index effecting the vehicle's handling and stability.

\section{Conclusion}

Aiming at finding a reasonable method of the large inertia vehicle s evaluation of handling and stability performance, relying on three road tests, this paper obtained 8 groups of test data about evaluation index, and obtained 3 groups of weighting coefficients by using three different objective weighting methods: variation coefficient method, entropy method and correlation coefficient method. An improved D-S evidence theory was raised and used to fuse the three weighting methods. The fused result was compared with the result of subjective evaluation. The final results showed that the improved method of this paper could solve the conflict between three weighting methods, and was similar with human's subjective evaluation. The evaluation method in this paper showed great superiority in evaluating the large inertia vehicle's handling and stability reasonably, and also provided a reference for evaluation of large inertia vehicle s handling and stability.

\section{Acknowledgements}

This work was financially supported by the National Natural Science Foundation of China (51475462)

\section{References}

[1] LUKOWSKI S, MOMOT M, KRAEMER D, et al.(2009). Basic linear theory of handling and stability of automobiles. Proceedings of the Institution of Mechanical Engineers Part D: Journal of Automobile Engineering, 223, 1-10. 
[2] FRENDO F, GRECO G, GUIGGIANI M, et al. (2008). Evaluation of the vehicle handling performances by a new approach. Vehicles system dynamics: International Journal of Vehicle Mechanics and Mobility, 46, 857-868.

[3] ZSCHOCKE A, ALBERS A. (2008). Links between subjective and objective evaluations regarding the steering character of automobiles. International Journal of Automotive Technology, 9, 473-481.

[4] WANG Yan-hui, GUO Kong-hui, LU Bing-wu, et al. (2011). Method of the vehicle's handling and stability objective evaluation based on multi-variate statistical analysis. Journal of Jilin University(Engineering and Technology Edition), 41, 6-11.

[5] HUANG Jian-xing, ZHAO You-qun. (2010). Comprehensive evaluation of human-vehicle closed-loop system for handling stability and technology of virtual prototype. Journal of Mechanical Engineering, 46, 102-108.

[6] XING Ru-fei, GUAN Xin, TIAN Cheng-wei, et al. (2009). Weight of subjective evaluation indexes for car handling and stability. Journal of Jilin University(Engineering and Technology Edition, 39, 33-38.

[7] GUO Kong-hui, JIN Ling-ge, CAO Yu, et al. (2010). Dimensional reduction of objective evaluation criteria of vehicle handling behavior. Automobile Technology, 2,1-4.

[8] YANG Yu. (2006). Analysis of weighting method of multi-index comprehensive evaluation. Statistics and Decision,7, 17-19.

[9] YU Xiao-fen, FU Dai.(2004). Overview of multi-index comprehensive evaluation method. Statistics and Decision, 11,119-121.

[10] AN Xiang-hua, FENG Yi-xiong, TAN Jian-rong. (2012). Collaborative evaluation method for product concept based on choquet integral and evidence theory. Journal of Zhejiang University(Engineering science, 46, 163-169.

[11] SUN Rui, HUANG Hong-zhong, MIAO Qiang. (2008). Improved information fusion approach based on D-S evidence theory. Journal of Mechanical Science and Technology, 22, 2417-2425.

[12] Liu W R .Analyzing the Degree of Conflict among Belief Functions[J] .Artificial Intelligence, 2006, 170(11): 909-924.

[13] QC/T 480-1999. (1999). Vehicle's handling and stability index limits and evaluation method. the State Quality Inspection Administration of China. 